\title{
Limit Circle/Limit Point Criteria for Second-Order Superlinear Differential Equations with a Damping Term
}

\author{
Lihong Xing, ${ }^{1}$ Wei Song, ${ }^{2}$ Zhengqiang Zhang, ${ }^{1}$ and Qiyi $\mathrm{Xu}^{1}$ \\ ${ }^{1}$ School of Electrical Engineering and Automation, Qufu Normal University, Rizhao, \\ Shandong 276826, China \\ ${ }^{2}$ The Thirteen Middle School of Jining, Jining 22100, China \\ Correspondence should be addressed to Lihong Xing, lhxingqfnu@126.com
}

Received 6 September 2011; Revised 13 December 2011; Accepted 18 December 2011

Academic Editor: PGL Leach

Copyright (C) 2012 Lihong Xing et al. This is an open access article distributed under the Creative Commons Attribution License, which permits unrestricted use, distribution, and reproduction in any medium, provided the original work is properly cited.

The purpose of the present paper is to establish some new criteria for the classifications of superlinear differential equations as being of the nonlinear limit circle type or of the nonlinear limit point type. The criteria presented here generalize some known results in literature.

\section{Introduction}

In 1910, Weyl [1] published his now classic paper on eigenvalue problems for second-order linear differential equations of the form

$$
\left(a(t) y^{\prime}\right)^{\prime}+r(t) y=\theta y, \quad \theta \in C
$$

He classified this equation to be of the limit circle type if each solution $y(t)$ is square integrable (belongs to $L^{2}$ ), that is,

$$
\int_{0}^{\infty} y^{2}(t) d t<\infty
$$

and to be of the limit point type if at least one solution $y(t)$ does not belong to $L^{2}$, that is,

$$
\int_{0}^{\infty} y^{2}(t) d t=\infty
$$


He showed that the linear equation (1.1) always has at least one square integrable solution if $\operatorname{Im} \theta \neq 0$. Thus, for second-order linear equations with $\operatorname{Im} \theta \neq 0$, the problem reduces to whether (1.1) has one (limit point type) or two (limit circle type) square integrable solutions. This is known as the Weyl Alternative. Weyl also proved that if (1.1) is of the limit circle type for some $\theta_{0} \in C$, then it is of the limit circle type for all $\theta \in C$. In particular, this is true for $\theta=0$; that is, if we can show that

$$
\left(a(t) y^{\prime}\right)^{\prime}+r(t) y=0
$$

is of limit circle type, then (1.1) is of the limit circle type for all values of $\theta$. There is considerable interest in this problem over the years. The classification is important in spectral theory for linear equation (1.1) since it characterizes the number boundary conditions for differential operator generated by (1.1) being a self-adjoint operator. The analogous problem for nonlinear equations is relatively new and not as extensively studied as the linear cases. For a survey of known results on the linear and nonlinear problems as well as their relationships to other properties of solutions such as boundedness, oscillation, and convergence to zero, we refer the readers to [2-9] and the recent monograph [10]. In this paper, we will discuss the equation with a damping term:

$$
\left(a(t) y^{\prime}\right)^{\prime}+p(t) y^{\prime}+r(t) y^{2 k-1}=0,
$$

where $a, r: R_{+} \mapsto R$ and $p: R_{+} \mapsto R_{+}$are continuous, $a^{\prime}, r^{\prime} \in A C_{\mathrm{loc}}\left(R_{+}\right), a^{\prime \prime}, r^{\prime \prime} \in L_{\mathrm{loc}}^{2}\left(R_{+}\right)$, $a(t)>0, r(t)>0$, and $k>0$ is a positive integer. When $p(t) \equiv 0$, then (1.5) turns into

$$
\left(a(t) y^{\prime}\right)^{\prime}+r(t) y^{2 k-1}=0,
$$

which is widely researched by many authors (see [10] and references cited therein).

Definition 1.1 (see [2]). A nontrivial solution $y(t)$ of (1.5) is said to be of the nonlinear limit circle type if

$$
\int_{0}^{\infty} y^{2 k}(t) d t<\infty
$$

and it is of the nonlinear limit point type otherwise, that is,

$$
\int_{0}^{\infty} y^{2 k}(t) d t=\infty
$$

Equation (1.5) is said to be of the nonlinear limit circle type if all its solutions satisfy (1.7), and it is said to be of the nonlinear limit point type if there is at least one nontrivial solution satisfying (1.8).

In this paper, we will give necessary and sufficient conditions to guarantee the nonlinear limit circle type or nonlinear point type for (1.5). 


\section{Main Results}

To simplify notations, let $\alpha=1 / 2(k+1)$, and $\beta=(2 k+1) / 2(k+1)$. We make the transformation

$$
s=\int_{0}^{t}\left[\frac{r^{\alpha}(u)}{a^{\beta}(u)}\right] d u, \quad x(s)=y(t) .
$$

Then (1.5) becomes

$$
\ddot{x}+A(t) \dot{x}+B(t) x^{2 k-1}=0,
$$

where $A(t)=p(t) / a^{\alpha}(t) r^{\alpha}(t)+\alpha\left((a(t) r(t))^{\prime} / a^{\alpha}(t) r^{\alpha+1}(t)\right)$, and $B(t)=(a(t) r(t))^{\beta-\alpha}$. Moreover, (2.2) can be rewritten as the system

$$
\dot{x}=z-A(t) x, \quad \dot{z}=\dot{A}(t) x-B(t) x^{2 k-1} .
$$

We are now ready to prove the first result for system (2.3).

Theorem 2.1. Assume that

$$
\begin{gathered}
\int_{0}^{\infty} \frac{A^{\prime}(u)}{B^{1 / 2}(u)} d u<\infty, \\
\int_{0}^{\infty} A^{\prime}(u) B^{1 / 2}(u) d u<\infty, \\
\int_{0}^{\infty} \frac{1}{B(u)} d u<\infty .
\end{gathered}
$$

Then (1.5) is of the nonlinear limit circle type; that is, any solution $y(t)$ of (1.5) satisfies

$$
\int_{0}^{\infty} y^{2 k}(t) d t<\infty
$$

Proof. Define

$$
V(x, z, s)=\frac{z^{2}}{2}+B(t) \frac{x^{2 k}}{2 k}+\int_{0}^{t} p(\xi) \frac{r^{\beta-\alpha}(\xi)}{a^{2 \alpha}(\xi)} y^{2 k}(\xi) d \xi
$$

Then we have

$$
\begin{aligned}
\dot{V}= & z \dot{z}+\left[B(t) \frac{x^{2 k}}{2 k}\right]+p(t)[a(t) r(t)]^{\beta-2 \alpha} y^{2 k}(t) \\
= & z\left[\dot{A}(t) x-B(t) x^{2 k-1}\right]+B(t) x^{2 k-1} \dot{x}+\dot{B}(t) \frac{x^{2 k}}{2 k} \\
& +p(t)[a(t) r(t)]^{\beta-2 \alpha} y^{2 k}(t)
\end{aligned}
$$




$$
\begin{aligned}
= & \dot{A}(t) x z-B(t) x^{2 k-1} z+B(t) x^{2 k-1}[z-A(t) x] \\
& +\dot{B}(t) \frac{x^{2 k}}{2 k}+p(t)[a(t) r(t)]^{\beta-2 \alpha} y^{2 k}(t) \\
= & \dot{A}(t) x z-A(t) B(t) x^{2 k}+\dot{B}(t) \frac{x^{2 k}}{2 k}+p(t)[a(t) r(t)]^{\beta-2 \alpha} y^{2 k}(t) \\
= & \dot{A}(t) x z+\left[\frac{\dot{B}(t)}{2 k}-A(t) B(t)\right] x^{2 k}+p(t)[a(t) r(t)]^{\beta-2 \alpha} y^{2 k}(t) \\
= & \dot{A}(t) x z-p(t)[a(t) r(t)]^{\beta-2 \alpha} x^{2 k}(s)+p(t)[a(t) r(t)]^{\beta-2 \alpha} y^{2 k}(t) \\
= & \dot{A}(t) x z .
\end{aligned}
$$

Since $|x z|=\left|B^{1 / 2}(t) x z / B^{1 / 2}(t)\right| \leq\left(B(t) x^{2} / 2+z^{2} / 2\right) / B^{1 / 2}(t) \leq\left(B(t)\left[x^{2} / 2 k+K_{1}\right]+\right.$ $\left.z^{2} / 2\right) / B^{1 / 2}(t) \leq V(s) / B^{1 / 2}(t)+K_{1} B^{1 / 2}(t)$ for some constant $K_{1} \geq 0$, we have

$$
\dot{V}(s) \leq \frac{|\dot{A}(t)| V(s)}{B^{1 / 2}(t)}+K_{1}|\dot{A}(t)| B^{1 / 2}(t) .
$$

Since

$$
\dot{A}(t)=A^{\prime}(t) \frac{d t}{d s}=A^{\prime}(t) \frac{a^{\beta}(t)}{r^{\alpha}(t)}
$$

letting $\tau(s)$ denote the inverse function of $s(t)$, we obtain

$$
\int_{0}^{s} \frac{|\dot{A}(\tau(v))|}{B^{1 / 2}(\tau(v))} d v=\int_{0}^{t} \frac{A^{\prime}(u)}{B^{1 / 2}(u)} d u
$$

Moreover,

$$
\int_{0}^{s}|\dot{A}(\tau(v))| B^{1 / 2}(\tau(v)) d v=\int_{0}^{t} A^{\prime}(u) B^{1 / 2}(u) d u
$$

Integrating (2.10) from 0 to $s$, we obtain

$$
V(s) \leq V(0)+\int_{0}^{s} \frac{|\dot{A}(\tau(v))|}{B^{1 / 2}(\tau(v))} V(v) d v+K_{1} \int_{0}^{s}|\dot{A}(\tau(v))| B^{1 / 2}(\tau(v)) d v .
$$

Condition (2.5) implies that the second integral on the right-hand side of (2.10) is convergent. By Gronwall's inequality, we have

$$
V(s) \leq M_{1} \exp \int_{0}^{s} \frac{|\dot{A}(\tau(v))|}{B^{1 / 2}(\tau(v))} d v
$$


for some constant $M_{1}>0$. By condition (2.4), the aforementioned integral is convergent, and we have that $V(s)$ is bounded, say, $V(s) \leq M_{2}$ for some $M_{2}>0$. Therefore,

$$
B(t) y^{2 k}(t)=B(t) x^{2 k}(s) \leq 2 k M_{2}
$$

from which it follows that

$$
\int_{0}^{\infty} y^{2 k}(u) d u \leq 2 k M_{2} \int_{0}^{\infty} \frac{1}{B(u)} d u<\infty
$$

by condition (2.6), so all solutions of (1.5) are of the nonlinear limit circle type, and this completes the proof of Theorem 2.1.

If $a(t) \equiv 1$ in (1.5), then it turns into

$$
y^{\prime \prime}+p(t) y^{\prime}+r(t) y^{2 k-1}=0,
$$

and we have the following corollary.

Corollary 2.2. Assume that

$$
\begin{gathered}
\int_{0}^{\infty} \frac{\left|\left[p(u) / r^{\alpha}(u)+\alpha r^{\prime}(u) / r^{1+\alpha}(u)\right]^{\prime}\right|}{r^{(\beta-\alpha) / 2}(u)} d u<\infty, \\
\int_{0}^{\infty}\left|\left[\frac{p(u)}{r^{\alpha}(u)}+\frac{\alpha r^{\prime}(u)}{r^{1+\alpha}(u)}\right]^{\prime}\right| r^{(\beta-\alpha) / 2}(u) d u<\infty, \\
\int_{0}^{\infty} \frac{1}{r^{\beta-\alpha}(u)} d u<\infty
\end{gathered}
$$

Then (2.18) is of the nonlinear limit circle type.

The aforementioned theorem and corollary offer sufficient conditions to guarantee (1.5) and (2.18) to be of the nonlinear limit circle type, respectively. The next theorem gives necessary conditions to guarantee that (1.5) is of the nonlinear limit circle type.

Lemma 2.3 (see [10]). Assume that there exists $N_{1}>0$ such that

$$
\begin{gathered}
\left|\frac{[a(t) r(t)]^{\prime}}{a^{1 / 2}(t) r^{3 / 2}(t)}\right| \leq N_{1}, \\
\int_{0}^{\infty} \frac{\left[(a(t) r(t))^{\prime}\right]^{2}}{a(t) r^{3}(t)} d t<\infty .
\end{gathered}
$$


If $y(t)$ is a nonlinear limit circle type solution of (1.5), then

$$
\int_{0}^{\infty} \frac{\left[(a(t) r(t))^{\prime}\right]^{2} y^{2}(t)}{a(t) r^{3}(t)} d t<\infty
$$

Proof. We have

$$
\int_{0}^{\infty} \frac{\left[(a(t) r(t))^{\prime}\right]^{2} y^{2}(t)}{a(t) r^{3}(t)} d t \leq N_{1}^{2} \int_{0}^{\infty} y^{2 k}(t) d t+\int_{0}^{\infty} \frac{\left[(a(t) r(t))^{\prime}\right]^{2}}{a(t) r^{3}(t)} d t<\infty
$$

by (1.7) and (2.21).

Theorem 2.4. Suppose that there exist constants $N_{2}>0$ and $M_{3}>0$ such that

$$
\begin{gathered}
\left|\frac{a^{1 / 2}(t) r^{\prime}(t)}{r^{3 / 2}(t)}\right| \leq N_{2}, \\
\int_{0}^{\infty} \frac{a(u)\left[r^{\prime}(u)\right]^{2}}{r^{3}(u)} d u<\infty, \\
\frac{p^{2}(t)}{a(t) r(t)} \leq M_{3}, \\
\int_{0}^{\infty} \frac{p^{2}(t)}{a(t) r(t)} d t<\infty .
\end{gathered}
$$

If $y(t)$ is a nonlinear limit circle type solution of (1.5), then

$$
\int_{0}^{\infty} \frac{a(u)\left[y^{\prime}(u)\right]^{2}}{r(u)}<\infty
$$

Proof. If we multiply (1.5) by $y(t) / r(t)$, use the identity

$$
\left(a(t) y^{\prime}\right)^{\prime}=\left(a(t) y^{\prime} y\right)^{\prime}-a(t)\left[y^{\prime}\right]^{2},
$$

and to integrate by parts, we obtain

$$
\begin{aligned}
& \frac{a(t) y^{\prime}(t) y(t)}{r(t)}-\frac{a\left(t_{1}\right) y^{\prime}\left(t_{1}\right) y\left(t_{1}\right)}{r\left(t_{1}\right)}+\int_{t_{1}}^{t} \frac{a(u) y^{\prime}(u) y(u) r^{\prime}(u)}{r^{2}(u)} d u \\
& +\int_{t_{1}}^{t} y^{2 k}(u) d u+\int_{t_{1}}^{t} \frac{p(u) y^{\prime}(u) y(u)}{r(u)} d u-\int_{t_{1}}^{t} \frac{a(u)\left[y^{\prime}(u)\right]^{2}}{r(u)} d u=0
\end{aligned}
$$


Journal of Applied Mathematics

for any $t_{1} \geq 0$. Now conditions (2.26), (2.27), and (1.7) imply

$$
\int_{t_{1}}^{\infty} \frac{p^{2}(u)\left[y^{2 k}(u)+1\right]}{a(u) r(u)} d u \leq M_{3} \int_{t_{1}}^{\infty} y^{2 k}(u) d u+\int_{t_{1}}^{\infty} \frac{p^{2}(u)}{a(u) r(u)} d u<\infty .
$$

Therefore, there exists a constant $k_{0}>0$, subject to

$$
\int_{t_{1}}^{t} \frac{p^{2}(u)\left[y^{2 k}(u)+1\right]}{a(u) r(u)} d u \leq M_{3} \int_{t_{1}}^{t} y^{2 k}(u) d u+\int_{t_{1}}^{t} \frac{p^{2}(u)}{a(u) r(u)} d u<k_{0}^{2}
$$

Applying Schwartz inequality, we obtain

$$
\begin{aligned}
\int_{t_{1}}^{t} \frac{p(u) y^{\prime}(u) y(u)}{r(u)} d u & \leq\left(\int_{t_{1}}^{t} \frac{a(u)\left[y^{\prime}(u)\right]^{2}}{r(u)} d u\right)^{1 / 2}\left(\int_{t_{1}}^{t} \frac{p^{2}(u)\left[y^{2 k}(u)+1\right]}{a(u) r(u)} d u\right)^{1 / 2} \\
& \leq k_{0}\left(\int_{t_{1}}^{t} \frac{a(u)\left[y^{\prime}(u)\right]^{2}}{r(u)} d u\right)^{1 / 2} .
\end{aligned}
$$

By the Schwartz inequality,

$$
\int_{t_{1}}^{t} \frac{a(u) y^{\prime}(u) y(u) r^{\prime}(u)}{r^{2}(u)} d u \leq\left[\int_{t_{1}}^{t} \frac{a(u)\left[y^{\prime}(u)\right]^{2}}{r(u)} d u\right]^{1 / 2}\left[\int_{t_{1}}^{t} \frac{a(u) y^{2}(u)\left[r^{\prime}(u)\right]^{2}}{r^{3}(u)} d u\right]^{1 / 2} .
$$

Now (2.24) implies

$$
\frac{a(t) y^{2}(t)\left[r^{\prime}(t)\right]^{2}}{r^{3}(t)} \leq \frac{a(t)\left[r^{\prime}(t)\right]^{2}}{r^{3}(t)}\left[y^{2 k}(t)+1\right] \leq N_{2}^{2} y^{2 k}(t)+\frac{a(t)\left[r^{\prime}(t)\right]^{2}}{r^{3}(t)} .
$$

So integrating the previous inequality and applying (2.25) and (1.7), we obtain

$$
\int_{t_{1}}^{\infty} \frac{a(u) y^{2}(u)\left[r^{\prime}(u)\right]^{2}}{r^{3}(u)} d u \leq K_{2}^{2}<\infty
$$

for some constant $K_{2}>0$.

If $y(t)$ is not eventually monotonic, let $\left\{t_{j}\right\} \rightarrow \infty$ be an increasing sequence of zeros of $y^{\prime}(t)$. Then from (2.30), we have

$$
\left(k_{0}+K_{2}\right) H^{1 / 2}\left(t_{j}\right)+K_{3} \geq H\left(t_{j}\right),
$$

where

$$
H(t)=\int_{t_{1}}^{t} \frac{a(u)\left[y^{\prime}(u)\right]^{2}}{r(u)} d u,
$$


and $K_{3}>0$ is a constant. It follows that $H\left(t_{j}\right) \leq K_{4}<\infty$ for all $j$ and for some constant $K_{4}>0$, so (2.28) holds.

If $y(t)$ is eventually monotonic, then $y(t) y^{\prime}(t) \leq 0$ for all $t \geq t_{1}$ for sufficiently large $t_{1} \geq 0$ since otherwise (1.7) would be violated. Using this fact in (2.30), we can repeat the type of argument used previously to obtain that (2.28) holds.

The following theorem gives sufficient conditions to ensure that (1.5) is of the nonlinear limit point type.

Theorem 2.5. Suppose that conditions (2.4), (2.5), and (2.20)-(2.27) hold. If

$$
\int_{0}^{\infty} \frac{1}{B(u)} d u=\infty
$$

then (1.5) is of the nonlinear limit point type.

Proof. As in the proof of Theorem 2.1, define

$$
V(x, z, s)=\frac{z^{2}}{2}+B(t) \frac{x^{2 k}}{2 k}
$$

and differentiate it to obtain

$$
\dot{V} \geq-\frac{|\dot{A}(t)| V(s)}{B^{1 / 2}(t)}-K_{1}|\dot{A}(t)| B^{1 / 2}(t),
$$

and so we then have

$$
\dot{V}+\frac{|\dot{A}(t)| V(s)}{B^{1 / 2}(t)} \geq-K_{1}|\dot{A}(t)| B^{1 / 2}(t) .
$$

If we define functions $H$ and $h: R_{+} \mapsto R$ by $H(t)=|\dot{A}(t)| / B^{1 / 2}(t)$, and $h(t)=K_{1}|\dot{A}(t)| B^{1 / 2}(t)$, then we have

$$
\frac{d}{d s}\left(\exp \int_{0}^{s} H(\tau(\xi)) d \xi\right) \geq-h(t) \exp \int_{0}^{s} H(\tau(\xi)) d \xi
$$

Now condition (2.4) guarantees that

$$
\exp \int_{0}^{\infty} H(\tau(\xi)) d \xi \leq K_{5}<\infty
$$

for some constant $K_{5}>0$, while condition (2.5) implies that

$$
K_{5} \int_{0}^{\infty} h(\tau(\xi)) d \xi \leq K_{6}<\infty
$$


for some $K_{6}>0$. Let $y(t)$ be any solution of (1.5) such that $V(x(0), z(0), 0)>K_{6}+1$. Integrating (2.43), we get

$$
V(s) \exp \int_{0}^{s} H(\tau(\xi)) d \xi \geq V(0)-K_{6}>1
$$

and so

$$
V(s) \geq \frac{1}{K_{5}}
$$

for $s \geq 0$. Dividing both sides of this last inequality by $B(t)$ and rewriting the left-hand side in terms of $t$, we have

$$
\begin{aligned}
& \frac{a(t)\left[y^{\prime}(t)\right]^{2}}{2 r(t)}+\frac{y^{2 k}(t)}{2 k}+\left[\frac{p(t)}{r(t)}+\alpha \frac{(a(t) r(t))^{\prime}}{r^{2}(t)}\right] y(t) y^{\prime}(t) \\
& +\left[\frac{p^{2}(t)}{2 a(t) r(t)}+\alpha \frac{p(t)(a(t) r(t))^{\prime}}{a(t) r^{2}(t)}+\alpha^{2} \frac{\left[(a(t) r(t))^{\prime}\right]^{2}}{2 a(t) r^{3}(t)}\right] y^{2}(t) \geq \frac{1}{K_{5}[a(t) r(t)]^{\beta-\alpha}}
\end{aligned}
$$

If $y(t)$ is a limit circle type solution of (1.5), then Theorem 2.4 implies

$$
\int_{0}^{\infty} \frac{a(t)\left[y^{\prime}(t)\right]^{2}}{r(t)} d t<\infty
$$

and Lemma 2.3 implies

$$
\int_{0}^{\infty} \frac{\left[(a(t) r(t))^{\prime}\right]^{2} y^{2}(t)}{a(t) r^{3}(t)} d t<\infty
$$

By the Schwartz inequality,

$$
\left|\int_{0}^{\infty} \frac{(a(t) r(t))^{\prime}}{r^{2}(t)} y(t) y^{\prime}(t) d t\right| \leq\left[\int_{0}^{\infty} \frac{\left[(a(t) r(t))^{\prime}\right]^{2} y^{2}(t)}{a(t) r^{3}(t)} d t\right]^{1 / 2} \times\left[\int_{0}^{\infty} \frac{a(t)\left[y^{\prime}(t)\right]^{2}}{r(t)} d t\right]^{1 / 2}<\infty
$$

From condition (2.27), using Schwartz inequality, we get

$$
\int_{0}^{\infty} \frac{p(t)(a(t) r(t))^{\prime}}{a(t) r^{2}(t)} d t \leq\left[\int_{0}^{\infty} \frac{\left[(a(t) r(t))^{\prime}\right]^{2} y^{2}(t)}{a(t) r^{3}(t)} d t\right]^{1 / 2} \times\left[\int_{0}^{\infty} \frac{p^{2}(t)}{a(t) r(t)} d t\right]^{1 / 2}<\infty
$$


So that if $y(t)$ is a limit circle solution of (1.5), from conditions (2.26) and (2.27), we obtain

$$
\begin{gathered}
\int_{0}^{\infty} \frac{p^{2}(t) y^{2}(t)}{2 a(t) r(t)} d t<\int_{0}^{\infty} \frac{p^{2}(t)\left[y^{2 k}(t)+1\right]}{2 a(t) r(t)} d t<\infty, \\
\int_{0}^{\infty} \frac{\alpha p(t)(a(t) r(t))^{\prime} y^{2}(t)}{a(t) r^{2}(t)} d t \leq \alpha\left(\int_{0}^{\infty} \frac{p^{2}(t) y^{2}(t)}{a(t) r(t)} d t\right)^{1 / 2}\left(\int_{0}^{\infty} \frac{\left[(a(t) r(t))^{\prime}\right]^{2} y^{2}(t)}{a(t) r^{3}(t)} d t\right)^{1 / 2}<\infty .
\end{gathered}
$$

Furthermore,

$$
\int_{0}^{\infty} \frac{p(t) y^{\prime}(t) y(t)}{r(t)} d t \leq\left(\int_{0}^{\infty} \frac{a(t)\left[y^{\prime}(t)\right]^{2}}{r(t)} d t\right)^{1 / 2}\left(\int_{0}^{\infty} \frac{p^{2}(t)\left[y^{2}(t)\right]}{a(t) r(t)} d t\right)^{1 / 2}<\infty
$$

Consequently, integrating both sides of (2.48), we see that the integrand of the left side of (2.48) is bounded, but the integrand of the right side of (2.48) tends to infinity according to condition (2.39). This leads to a contradiction, so $y(t)$ is a limit point type solution of (1.5), and (1.5) is of the nonlinear limit point type.

The last theorem and corollary give the sufficient and necessary conditions to guarantee (1.5) and (2.18) to be of the nonlinear limit circle type, respectively.

Theorem 2.6. Assume that conditions (2.4), (2.5), (2.20), (2.21), (2.24), (2.25), (2.26), and (2.27) hold. Then (1.5) is of the nonlinear limit circle type if and only if

$$
\int_{0}^{\infty} \frac{1}{(a(t) r(t))^{k /(k+1)}} d t<\infty
$$

When one specializes this theorem to (2.18), that is, $a(t) \equiv 1$ in (1.5), one obtains the following corollary.

Corollary 2.7. Assume that

$$
\begin{array}{cc}
\int_{0}^{\infty} \frac{\left[p(t) / r^{\alpha}(t)+\alpha r^{\prime}(t) / r^{\alpha+1}(t)\right]^{\prime}}{r^{(\beta-\alpha) / 2}(t)} d t<\infty, \quad \int_{0}^{\infty}\left[\frac{p(t)}{r^{\alpha}(t)}+\frac{\alpha r^{\prime}(t)}{r^{\alpha+1}(t)}\right]^{\prime} r^{(\beta-\alpha) / 2}(t) d t<\infty, \\
\frac{\left|r^{\prime}(t)\right|}{r^{3 / 2}(t)} \leq N_{1}, \quad \int_{0}^{\infty} \frac{\left[r^{\prime}(t)\right]^{2}}{r^{3}(t)} d t<\infty, \quad \frac{p^{2}(t)}{r(t)} \leq M_{3}, \quad \int_{0}^{\infty} \frac{p^{2}(t)}{r(t)} d t<\infty .
\end{array}
$$

Then (2.18) is of the nonlinear limit circle type if and only if

$$
\int_{0}^{\infty} \frac{1}{r(t)^{k /(k+1)}} d t<\infty
$$




\section{Acknowledgments}

The authors would like to thank the referee for his helpful comments on this paper. This research was partially supported by the NSF of China (Grant 61104007) and NSF of Qufu Normal University (Grant xj201023).

\section{References}

[1] H. Weyl, "Über gewöhnliche differentialgleichungen mit singularitäten und die zugehörigen entwicklungen willkürlicher funktionen," Mathematische Annalen, vol. 68, no. 2, pp. 220-269, 1910.

[2] M. Bartušek, Z. Doslá, and J. R. Graef, "On the definations of the nonlinear limit-point/limit-circle properties," Differential Equations and Dynamical Systems, vol. 9, pp. 49-61, 2001.

[3] J. R. Graef, "Limit circle type criteria for nonlinear differential equations," Proceedings of the Japan Academy, Series A, vol. 55, no. 2, pp. 49-52, 1979.

[4] J. R. Graef, "Limit circle criteria and related properties for nonlinear equations," Journal of Differential Equations, vol. 35, no. 3, pp. 319-338, 1980.

[5] J. R. Graef and P. W. Spikes, “On the nonlinear limit-point/limit-circle problem," Nonlinear Analysis, vol. 7, no. 8, pp. 851-871, 1983.

[6] P. W. Spikes, "Criteria of limit circle type for nonlinear differential equations," SIAM Journal on Mathematical Analysis, vol. 10, no. 3, pp. 456-462, 1979.

[7] M. V. Fedoryuk, Asymptotic Analysis, Springer, Berlin, Germany, 1993.

[8] P. Hartman and A. Wintner, "Criteria of non-degeneracy for the wave equation," American Journal of Mathematics, vol. 70, pp. 295-308, 1948.

[9] M. Bartušek and J. R. Graef, "The nonlinear limit-point/limit-circle problem for second order equations with p-Laplacian," Dynamic Systems and Applications, vol. 14, no. 3-4, pp. 431-446, 2005.

[10] M. Bartušek, Z. Doslá, and J. R. Graef, "The nonlinear limitpoint/limit-circle problem," Birkhauser, vol. 3, pp. 34-35, 2005. 


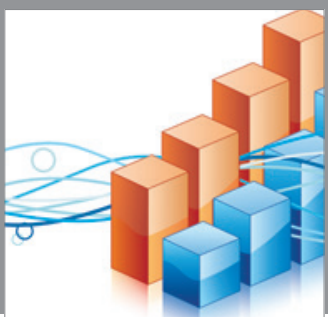

Advances in

Operations Research

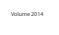

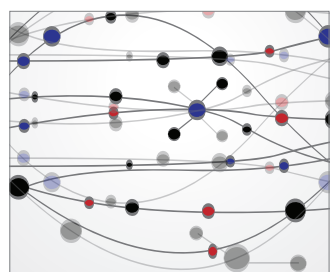

\section{The Scientific} World Journal
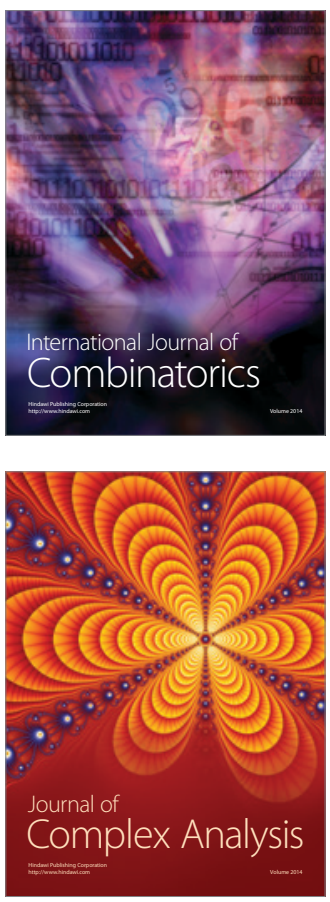

International Journal of

Mathematics and

Mathematical

Sciences
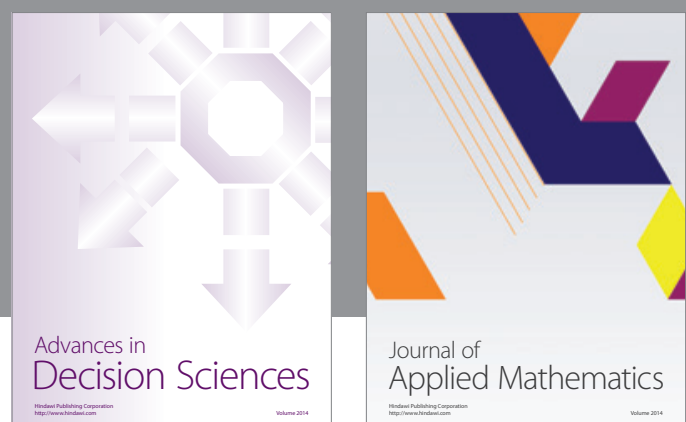

Journal of

Applied Mathematics
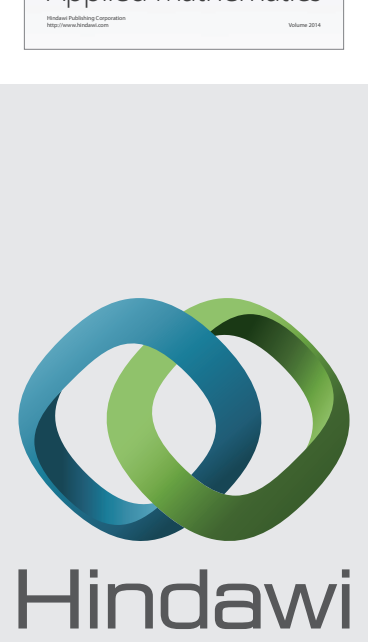

Submit your manuscripts at http://www.hindawi.com
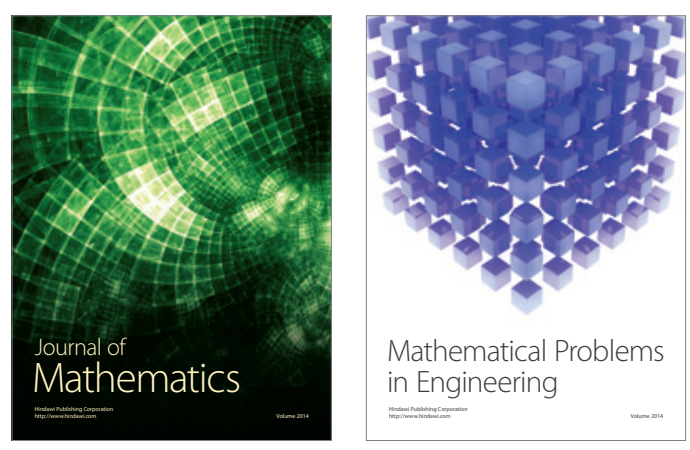

Mathematical Problems in Engineering
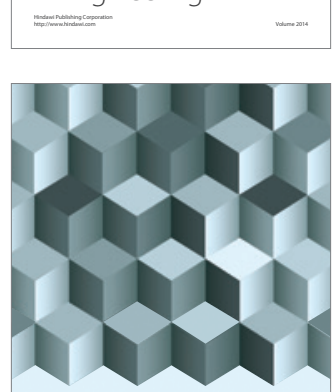

Journal of

Function Spaces
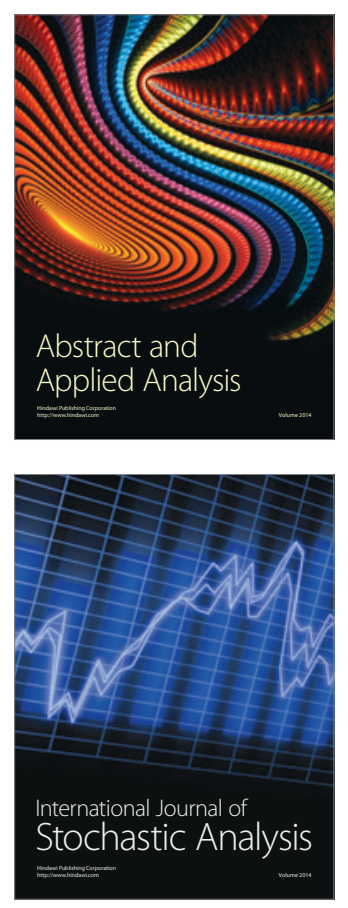

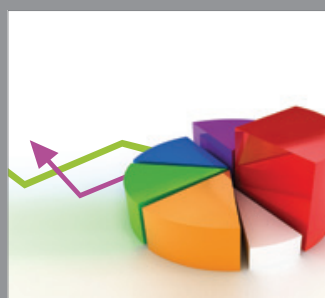

ournal of

Probability and Statistics

Promensencen
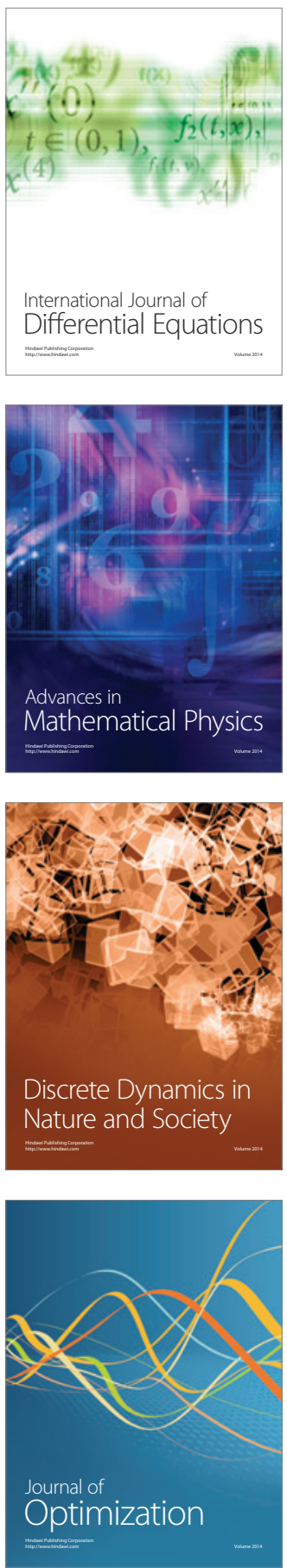